\title{
Perceptual specificity of priming for compound words not presented
}

\author{
TODD C. JONES \\ Victoria University of Wellington, Wellington, New Zealand
}

\begin{abstract}
Two experiments extended prior work on the perceptual specificity of priming to compound words not presented during a study phase. In both experiments, perceptual manipulations were employed, and priming was obtained on a word fragment completion test (e.g., $c \_e c \_p o \_n t$ ) where the lexical elements of the compounds (check and point) were presented in different study words. In Experiment 1, priming was highest when identical fragments for the lexical components were presented during the study phase (c_ec_list, needlepo_nt) and test phase (c_ec_po_nt). In Experiment 2, visual study presentations, but not auditory study presentations, led to significant priming. The findings are consistent with predictions from transfer-appropriate processing and demonstrate perceptual, not lexical (postperceptual), priming.
\end{abstract}

In the present experiments, a word fragment completion test was used to examine priming for compound words not presented during the study phase. To achieve this small feat, parent compound words (e.g., checklist, needlepoint) were presented during the study phase. On a later word fragment completion test, lexical components of the study words were rearranged to create conjunction words (checkpoint) that had not been presented in their whole form during the study phase (cf. Reinitz \& Demb, 1994). Below, prior results and theory on conjunction word priming are described briefly, and a different theoretical approach is offered.

Reinitz and Demb (1994) found significant conjunction word priming on a word fragment completion test but not two perceptual identification tests (see Reinitz \& Alexander, 1996, for another null result on perceptual identification). Priming scores were always significantly lower for conjunction words than for old words. Also, the pattern of performance for word fragment completion was similar to that for recognition memory. The authors proposed that independent access of postperceptual representations for stimulus parts (e.g., check from checklist and point from needlepoint) supports performance on word fragment completion and recognition memory tasks, but not on perceptual identification tasks. However, significant perceptual identification priming for conjunction words and feature words (half old, half new words) has since been obtained (Reinitz, Verfaellie, \& Milberg, 1996). This finding appears to be at odds with the earlier theoretical proposal: If priming for conjunction words can be obtained

A School of Psychology Research Grant provided funding. I thank Stefanie Sharman and Kirsty Novis for data collection assistance and Lisa Geraci, Kathleen McDermott, Neil Mulligan, and Roddy Roediger for helpful comments. Correspondence should be addressed to T. C. Jones, School of Psychology, Victoria University of Wellington, Box 600, Wellington, New Zealand (e-mail: todd.jones@ vuw.ac.nz). for perceptual identification, too, then additional representations seem unnecessary.

An alternative is that different types of familiarity (or automatic processes) contribute to performance on recognition and perceptual implicit tests (Wagner, Gabrieli, \& Verfaellie, 1997; see also Jones, Jacoby, \& Gellis, 2001). Of particular importance here is the idea that perceptual familiarity drives performance on perceptual implicit tests (for a review, see Roediger \& McDermott, 1993). However, it has also been suggested that lexical processing provides some contribution to performance on perceptual implicit tests (Richardson-Klavehn \& Gardiner, 1998; Weldon, 1991).

One possibility is that lexical processing of the separate constituents in a compound word leads to priming of conjunction words. (This idea is consistent with Reinitz \& Demb's, 1994, proposal of postperceptual representations of stimulus parts.) To date, the priming of conjunction words has not been assessed with a perceptual manipulation. In the present experiments, perceptual manipulations were used to evaluate whether the familiarity underlying conjunction word priming is perceptual or lexical (or both).

Experiment 1 centered on perceptual specificity results obtained by Gardiner (1988) and Gardiner, Dawson, and Sutton (1989). Gardiner (1988) found priming for generated and read words (e.g., hired political killer: $a \_s \_s \_i n$ or hired political killer: assassin), but a specificity effect was obtained such that generating a word from an identical fragment at study and test $\left(a \_s \_s \_i n\right)$ produced greater priming than did reading a word or generating a word with a different perceptual cue ( $a s \_a \_s \_n$ ). This manipulation was employed at the constituent level of compound words instead of the whole word. Transfer-appropriate processing theory (Roediger, Weldon, \& Challis, 1989) predicts that priming should be highest in the condition where there is the greatest overlap in perceptual cues from study 
to test (a perceptual familiarity hypothesis). Thus, if priming were to be obtained at all, it should be in a condition where the fragmented portions of a test stimulus matched those from portions of earlier study stimuli. In contrast, if postperceptual, lexical processing is wholly responsible for conjunction word priming, then no perceptual specificity effect should be observed. Experiment 2 tested the possibility that postperceptual, lexical processing contributed to priming in Experiment 1 through cross-modal priming.

\section{EXPERIMENT 1}

On each study trial, letter information was deleted from one of the lexical constituents of a compound word, while the other lexical constituent was presented intact. Thus, each study trial involved word generation. The lexical constituents that were to be conjoined to produce the target words on the test were either read intact or generated, and there were two generation conditions. (Examples are shown in Table 1.) For one condition, the study fragments differed from those presented in the test fragment. For the other condition, the study fragments were identical to those presented in the test fragment. These three study conditions will be referred to as the intact, differentfragments, and same-fragments conditions, respectively. Because findings of priming for conjunction words have not been obtained consistently, one question was whether the study conditions would show higher completion rates than a nonstudied condition (a priming effect). The chief interest was whether the same-fragments condition would produce a higher completion rate than the intact and different-fragments conditions (a perceptual specificity effect). A minor point addressed was whether the differentfragments condition would produce a higher completion rate than the read condition (a more general generation effect and potential evidence of explicit contamination) or vice versa (a reverse generation effect). The perceptual familiarity hypothesis embedded in transfer-appropriate processing predicts that a same-fragments condition should show the highest level of priming. A postperceptual, lexical processing hypothesis predicts that priming should not differ across conditions.

To anticipate, the results of a pilot experiment showed significant priming for conjunction words in general, providing evidence for the lexical processing hypothesis. These data appear in Table 2. However, the same-fragments condition was significantly different from the intact and different-fragments conditions after items with extremely

Table 1

Example Stimuli for the Four Conditions

\begin{tabular}{llc}
\hline \multicolumn{1}{c}{ Condition } & Study Stimuli (Parents) & Test Stimulus \\
\hline Same-fragments & c_ec_list, needlepo_nt & c_ec_po_nt \\
Different-fragments & ch__klist, needlep_int & c_ec_po_nt \\
Intact & checkli_t, nee_lepoint & c_ec_po_nt \\
Nonstudied & \multicolumn{2}{c}{ c_ec_po_nt } \\
\hline
\end{tabular}

Table 2

Mean Completion Rates for a Pilot Experiment (for All Items and $75 \%$ of Items) and Experiment 1 by Condition

\begin{tabular}{|c|c|c|c|}
\hline \multirow[b]{2}{*}{ Condition } & \multicolumn{2}{|c|}{ Pilot Experiment } & \multirow{2}{*}{$\begin{array}{c}\text { Experiment } 1 \\
\text { (All Items) }\end{array}$} \\
\hline & All Items & $75 \%$ of Items & \\
\hline Same fragments & .69 & .65 & $.54 \quad(.59)$ \\
\hline Different fragments & .62 & .54 & $(.49)$ \\
\hline Intact & .64 & .57 & .42 \\
\hline Nonstudied & .52 & .39 & .31 \\
\hline
\end{tabular}

Note-The $75 \%$ of items in the pilot experiment was obtained by removing items in the highest quartile of nonstudied completion rates (e.g., ceiling effects). Numbers in parentheses are conditionalized for successful generation during the study phase.

high baseline completion rates (.80 and higher) were eliminated from the analyses ( $25 \%$ of the items), providing evidence in favor of a perceptual processing hypothesis. In Experiment 1, a subset of the original items from the pilot experiment was used, and a few minor changes were made to the procedure (e.g., the test presentation was shortened).

\section{Method}

Subjects. Twenty-four Victoria University of Wellington (VUW) introductory psychology students participated and received credit toward a course requirement. One to 5 subjects were run at a time, with each subject seated at an individual computer station.

Materials and Equipment. Forty compound words were used as targets; 80 words served as parent words for these targets. Thirteen extra compound words were buffer or practice items. Each study word contained one intact and one fragmented lexical constituent. Two sets of test fragments for the critical constituents (A and B) were used. The test fragments were constructed so that letters were missing from both lexical constituents of the compound word target. Finally, digital sound files (WAV files) were made for each of the parent and buffer words in a female voice. The experiment was conducted on five IBM-compatible computers equipped with headphones and E-prime software (Schneider, Eschman, \& Zuccolotto, 2002a, 2002b).

Design and Procedure. Study condition was a within-subjects variable with four levels: intact, same-fragments and differentfragments, where the lexical components to be tested were generated during the study phase, and nonstudied. The parent stimuli had one or two letters missing from one of the lexical constituents and were meant to produce high generation rates. For the intact condition, the critical lexical constituents were presented intact but the remaining noncritical lexical constituent had one or two letters missing. For the same- and different-fragments conditions, the fragmented constituents of the parents during study were both either identical to or different from those presented in the recombined test fragments. The 40 target words were divided into four lists of 10 words. Each list served in each of the study conditions equally often across subjects. Fragment Sets A and B were counterbalanced for use as test fragments across subjects.

In a first phase, subjects attempted to generate 60 parent words, 4 primacy buffers, and 4 recency buffers. All stimuli were presented in 18-point Times Roman font. A single study order was constructed on the basis of study condition, and items from the different lists were called into these condition slots according to a counterbalancing scheme. The three studied conditions were distributed evenly throughout the list, and the parent containing the second lexical constituent of a conjunction word was always presented first. The subjects were told that they were to try to solve the word fragments and 
that the interest was not how many fragments they could solve, but which fragments they could solve. On each study trial, a fragmented word was presented for $5 \mathrm{sec}$, followed by a 500-msec period of blank screen, a 1.4-sec period for the auditory presentation of the stimulus, a 2.9-sec response interval, and a 1-sec re-presentation of the fragmented word. The auditory presentation ensured that subjects knew the target so that they could indicate whether the correct target was generated. During the response interval, subjects pressed one of two keys to indicate whether the correct word had been generated. The intertrial interval was $1 \mathrm{sec}$.

For the test, the subjects were told that they would attempt to generate words from word fragments but that the fragments would be relatively difficult and presented for less time. They were instructed to write their answers on an answer sheet and to guess with the first word that came to mind or to put an " $\mathrm{X}$ " in a blank if no word came to mind. On each trial, a tone was heard and followed by a 500-msec presentation of a focal point (6 plus signs), a 250 -msec period of blank screen, a 500-msec presentation of the test fragment, and a 7sec mask display (12 question marks). Answers were written during the 7-sec mask display, and the subjects were told not to change their answers. (Pilot work indicated that it was difficult to write a word down in the time allotted if writing was not initiated immediately after the mask appeared.) The screen was then cleared, and the next trial began. There were 45 test trials. Five initial trials were fillers (all new words) to allow practice with the procedure; the remaining 40 trials were critical trials. The same order of test items was used for all subjects.

\section{Results and Discussion}

The study generation rates were quite good. The rates for the same-fragments (.91) and different-fragments (.89) conditions were similar and slightly lower than that for the intact condition (.95). The completions of the test fragments were scored strictly. To ensure that the answers were not generated on the basis of a refinement from a first attempt, answers that had been obviously changed to become target words (e.g., "blowgum" to "blowgun") were not counted as correct. The mean completion rates for the four conditions are shown in Table 2. (For this experiment, as well as Experiment 2, analyses on the unconditionalizedand conditionalizedmeans led to the same conclusions. Only the analyses for the unconditionalized data are reported.) As can be seen, the three studied conditions produced completion rates above that for the nonstudied condition. The same-fragments condition produced a higher completion rate than did the read and different-fragments conditions. To test the specific hypotheses, a repeated measures analysis of variance (ANOVA) was conducted with planned Helmert contrasts. (Alpha was set at .05 for all tests.) The ANOVA gave a significant result $\left[F(3,66)=8.160, M S_{\mathrm{e}}=.025\right]$. For the Helmert contrasts, the new condition was compared with the other three conditions to assess priming $[F(1,22)=$ $\left.23.124, M S_{\mathrm{e}}=.024\right]$; the same-fragments condition was compared with the intact and different-fragments conditions to assess the specificity effect $[F(1,22)=5.920$, $\left.M S_{\mathrm{e}}=.048\right]$; and the read and different-fragments conditions were compared to identify a more general generation effect or a reverse generation effect $[F(1,22)=.294$, $\left.M S_{\mathrm{e}}=.051\right]$.

First, significant priming was obtained. Second, a specific generation effect was produced. Third, no unam- biguous evidence was obtained for a more general generation effect (different fragments $>$ intact) or a reverse generation effect (intact $>$ different fragments). (Although the conditionalized data showed a trend toward a general generation effect, the difference was not significant.) Thus, although the test stimuli were recombinations of compound words, the pattern of data was similar to that obtained by Gardiner (1988) and Gardiner et al. (1989). These data provide support for the perceptual familiarity hypothesis over the lexical familiarity hypothesis.

\section{EXPERIMENT 2}

In Experiment 1, subjects saw the critical lexical constituents intact or generated them from visual cues. In addition, each lexical constituent was subsequently presented aurally within its parent word. Thus, an auditory, and hence lexical, contribution to the priming effect in Experiment 1 cannot be ruled out. The second experiment was conducted to assess a potential lexical contribution to the priming effect via a cross-modal (auditory) presentation.

Cross-modal recognition conjunction errors have been obtained reliably (Jones et al., 2001). If postperceptual, lexical representations provide a basis for conjunction word priming, and if the same postperceptual representations support word fragment completion and recognition memory (see, e.g., Reinitz \& Demb, 1994), then access to the lexical constituents from an auditory presentation should produce priming. On the other hand, if perceptual processes underlie performance, then there should be little, if any, priming from the auditory presentation (e.g., Blum \& Yonelinas, 2001).

The condition with the least perceptual overlap from study to test, the generate different-fragments condition, was thought potentially to benefit the most from an auditory presentation. Thus, this condition represented the generate conditions in this second experiment. The components of the study trials from the first experiment were assessed by using three study presentation conditions: visual fragment, auditory (only), and visual fragment followed by auditory presentation. A comparison of the auditory condition and the nonstudied condition and a comparison of the visual fragment and the visual fragment plus auditory presentation condition provided evaluations of whether an auditory presentation might have contributed to priming in Experiment 1.

\section{Method}

Subjects. Thirty-two VUW introductory psychology students participated and received credit toward a course requirement. The computer stations were those used in Experiment 1.

Design and Procedure. There were four within-subjects study conditions: visual fragment, auditory, visual fragment plus auditory presentation, and nonstudied. The parent and target words and test fragments (counterbalanced across subjects) were those used in Experiment 1 .

The test procedure and the order of the items in the study and test trials were the same as those in Experiment 1. For the visual fragment plus auditory condition, the timing of events within a trial was the same as in Experiment 1 with the exception that the second vi- 
sual presentation was eliminated. For the visual fragment condition, there was a silence in place of the auditory presentation. For the auditory condition, there was a blank screen for $2.5 \mathrm{sec}$, followed by the auditory presentation in a 1.4-sec period, which was followed by $3 \mathrm{sec}$ of silence with a blank screen. To allow for responses on each study trial, including auditory only trials, the subjects indicated by a keypress whether the identity of a stimulus was clear to them within a $2.9-\mathrm{sec}$ period. The intertrial interval was $1 \mathrm{sec}$.

\section{Results and Discussion}

The proportion of "clear" judgments on the identity of the parents in the visual fragment and the visual fragment plus auditory conditions were .94 and .90 , respectively, demonstrating highly successful word generation. The test data, scored with the same strict criterion as that used in Experiment 1, are presented in Table 3. The visual fragment and visual fragment plus auditory conditions produced similar mean completion rates, and these completion rates were higher than those for the auditory and nonstudied conditions, which were similar to each other. A repeated measures ANOVA on the four conditions gave a significant result $\left[F(3,90)=8.83, M S_{\mathrm{e}}=.03\right]$, and a planned comparison of the visual conditions and the nonstudied condition showed that significant priming was obtained $\left[F(1,30)=19.19, M S_{\mathrm{e}}=.05\right]$.

To assess the contribution of an auditory presentation to priming, two planned comparisons were carried out. The first comparison indicated that the completion rate for the auditory condition was not significantly different from that for the nonstudied condition $\left[F(1,30)=0.63, M S_{\mathrm{e}}=\right.$ .06]. The second comparison showed that the completion rate for the visual fragment and visual fragment plus auditory presentation conditions were not significantly different $\left[F(1,30)=1.08, M S_{\mathrm{e}}=.05\right]$. Thus, there was no evidence that the auditory presentation, and hence lexical familiarity, contributed to the priming effect. (The auditoryonly condition was not compared with the visual fragment presentation conditions, because presentation modality was confounded with word generation.)

\section{GENERAL DISCUSSION}

The results from the two experiments show significant priming and perceptual specificity effects for compound words that had never been presented during the study phase. Previous research on conjunction words has produced significant priming on some occasions (Reinitz \& Demb, 1994, Experiment 3; Reinitz et al., 1996) but not

Table 3

Mean Completion Rates by Condition for Experiment 2

\begin{tabular}{lc}
\hline \multicolumn{1}{c}{ Condition } & Completion Rate \\
\hline Visual fragment + auditory presentation & $.52(.56)$ \\
Visual fragment & $.48(.53)$ \\
Auditory presentation & .37 \\
Nonstudied & .33 \\
\hline
\end{tabular}

Note-Numbers in parentheses are conditionalized for successful generation (a "clear" identity response) during the study phase. others (Reinitz \& Alexander, 1996; Reinitz \& Demb, 1994, Experiments 1 and 2). The present experiments add two new instances of significant word fragment completion priming for conjunction words to the single existing case (Reinitz \& Demb, 1994). The perceptual specificity effect demonstrates the importance of the overlap in perceptual information from study to test and extends the specificity of priming effects to the recombination of parts of previous stimuli (conjunction words). In addition, Experiment 2 did not provide evidence of any cross-modal contribution to the priming effect. Therefore, the effect appears to be strongly visual. These findings are consistent with expectations based on a perceptual familiarity hypothesis of transfer-appropriate processing theory but not a postperceptual, lexical familiarity (or representation) hypothesis. One possible explanation for the inconsistent priming effects of conjunction words on perceptual identification in other experiments may be that the viewing conditions of the task simply do not tightly constrain the possible targets relative to the conditions afforded in word fragment completion.

A novel aspect of the present results is that a change in context of the lexical elements from the parent words to the conjunction target does not appear to have been a large obstacle to achieving specificity effects. That is, in some sense the study and test stimuli were not identical, as they were in Gardiner and colleagues' (Gardiner, 1988; Gardiner et al., 1989) work, because the test fragments constituted the conjunction of components from previous stimuli. Shift in context is important-priming is much lower for conjunction words than for old words (Reinitz \& Alexander, 1996; Reinitz \& Demb, 1994; Reinitz et al., 1996)_priming can sometimes be obtained despite such a shift (also see Weldon's, 1991, Experiment 3). Subjects may have tried to solve the fragmented test constituents separately, but the brief test presentations would have made this strategy relatively difficult. Also, for this strategy, the absence of clearly demarcated constituents should have reduced completion rates. In Experiment 1, a small subset of test fragments whose constituents were not clearly demarcated (e.g., the final letter of the first constituent and the first letter of the second constituent were missing) produced results similar to those for other test fragments.

The specific generation effect obtained in the present experiments is highly unlikely to have been due to intentional retrieval strategies. First, the conjunction words themselves were never presented during the first phase, and no old words appeared on the test. Intentional retrieval strategies would probably have been helpful for retrieving parent words instead of the targeted conjunction words. In this case, such a strategy would have decreased the probability that target conjunction words would be produced. (Some answers given were parent words.) Second, the fragment cues were presented briefly $(500 \mathrm{msec})$ with a backward mask. Thus, relatively little time was given to view the test fragments. Finally, if somehow an intentional retrieval strategy could have been used to retrieve constituents of different parent words, then one might expect 
a significantly higher completion rate to be have been obtained for the different-fragments condition than for the read condition (a general generation effect). However, there was no clear evidence of a more general generation effect.

Neither Experiment 1 nor Gardiner and colleagues' (Gardiner, 1988; Gardiner et al., 1989) experiments have shown reverse generation effects (read intact $>$ generate different-fragments; for examples of reversals, see Roediger \& McDermott, 1993). The reason why these studies have not obtained reverse generation effects for intact versus generate different-fragments conditions may not be straightforward, reflecting one or a variety of methodological differences. For example, the difference in perceptual overlap from study to test for these conditions was relatively small, the constituents were presented in a context instead of in isolation, and the materials were different from those in other studies. Also, for the present study, the same effort should have been applied across the study conditions because generation of the study words occurred on each trial.

Finally, these findings are consistent with models of memory that assume distributed storage of representations and that assume simultaneous activation of list items during retrieval (e.g., an auto-associative model; Chappell \& Humphreys, 1994). The production of a conjunction word in the present study to some degree resembles the retrieval of a nonstudied word $(\mathrm{dog})$ in immediate category cued recall after the presentation of words that contain the phonemes of that word (dart, mop, and fig; Tehan \& Humphreys, 1998), though conceptual cues were absent in the present experiments. Thus, in some circumstances, emergent outcomes (i.e., blends or conjunctions) from interactive effects within some memory models could extend to priming phenomena (see also, e.g., Reinitz et al., 1996). One possible caveat for this suggestion is that significant priming for half-old compound words (e.g., checklist after studying checkpoint, Reinitz et al., 1996) would appear not to be accommodated with an emergent outcome explanation. However, such models seem worthy of consideration.

\section{REFERENCES}

Blum, D., \& Yonelinas, A. P. (2001). Transfer across modality in perceptual implicit memory. Psychonomic Bulletin \& Review, 8, 147-154.

Chappell, M., \& Humphreys, M. S. (1994). An auto-associative neural network for sparse representations: Analysis and application to models of recognition and cued recall. Psychological Review, 101, 103-128.

GARDINER, J. M. (1988). Generation and priming effects in wordfragment completion. Journal of Experimental Psychology: Learning, Memory, \& Cognition, 14, 495-501.

Gardiner, J. M., Dawson, A. S., \& Sutton, E. A. (1989). Specificity and generality of enhanced priming effects for self-generated study items. American Journal of Psychology, 102, 295-305.

JoNES, T. C., JACOBY, L. L., \& GELlis, L. A. (2001). Cross-modal feature and conjunction errors in recognition memory. Journal of Memory \& Language, 44, 131-152.

Reinitz, M. T., \& AlEXANDER, R. (1996). Mechanisms of facilitation in primed perceptual identification. Memory \& Cognition, 24, 129-135.

REINITZ, M. T., \& DEMB, J. B. (1994). Implicit and explicit memory for compound words. Memory \& Cognition, 22, 687-694.

Reinitz, M. T., Verfaellie, M., \& Milberg, W. P. (1996). Memory conjunction errors in normal and amnesic subjects. Journal of Memory \& Language, 35, 286-299.

Richardson-Klavehn, A., \& Gardiner, J. M. (1998). Depth-ofprocessing effects on priming in stem completion: Tests of the voluntary-contamination, conceptual-processing, and lexical-processing hypotheses. Journal of Experimental Psychology: Learning, Memory, \& Cognition, 24, 593-609.

RoEDiger, H. L., III, \& MCDERMOTT, K. B. (1993). Implicit memory in normal human subjects. In H. Spinnler \& F. Boller (Eds.), Handbook of neuropsychology (pp. 63-151). Amsterdam: Elsevier.

Roediger, H. L., III, Weldon, M. S., \& Challis, B. (1989). Explaining dissociations between implicit and explicit measures of retention: A processing account. In H. L. Roediger III \& F. I. M. Craik (Eds.), Varieties of memory and consciousness: Essays in honour of Endel Tulving (pp. 3-41). Hillsdale, NJ: Erlbaum.

SCHNeider, W., Eschman, A., \& Zuccolotto, A. (2002a). E-prime reference guide. Pittsburgh, PA: Psychology Software Tools.

Schneider, W., Eschman, A., \& Zuccolotto, A. (2002b). E-prime user's guide. Pittsburgh, PA: Psychology Software Tools.

Tehan, G., \& Humphreys, M. S. (1998). Creating proactive interference in immediate recall: Building a DOG from a DART, a MOP, and a FIG. Memory \& Cognition, 26, 477-489.

Wagner, A. D., Gabrieli, J. D. E., \& Verfaellie, M. (1997). Dissociations between familiarity processes in explicit recognition and implicit perceptual memory. Journal of Experimental Psychology: Learning, Memory, \& Cognition, 23, 305-323.

WELDON, M. S. (1991). Mechanisms underlying priming on perceptual tests. Journal of Experimental Psychology: Learning, Memory, \& Cognition, 17, 526-541.

(Manuscript received October 15, 2002;

revision accepted for publication January 15, 2003.) 